\title{
Severe Snakebite Envenoming in Intensive Care
}

\section{Jiří Valenta, Zdeněk Stach, Pavel Michálek}

Department of Anesthesiology and Intensive Care, First Faculty of Medicine, Charles University and General University Hospital in Prague, Prague, Czech Republic

Received September 14, 2016; Accepted November 14, 2016.

Key words: Snakebite - Exotic snakes - Envenoming - Treatment - Intensive care - Organ failure - Coagulopathy - Compartment syndrome

\begin{abstract}
Snakebites by exotic venomous snakes can cause serious or even lifethreatening envenoming. In Europe and North America most victims are breeders, with a few snakebites from wild native American rattlesnakes. The envenomed victims may present in organ and/or system failure with muscle paralysis, respiratory failure, circulatory instability, acute kidney injury, severe coagulation disorder, and local disability - compartment syndrome and necrosis. Best managed by close collaboration between clinical toxicology and intensive care, most severe envenomings are managed primarily by intensive care physicians. Due to the low incidence of severe envenoming, the clinical course and correct management of these cases are not intrinsically familiar to most physicians. This review article summarizes the clinical syndromes caused by severe envenoming and the therapeutic options available in the intensive care setting.
\end{abstract}

Mailing Address: Jiří Valenta, MD., Department of Anesthesiology and Intensive Care, First Faculty of Medicine, Charles University and General University Hospital in Prague, U Nemocnice 2, 12808 Prague 2, Czech Republic; Phone: +420 224962 248; e-mail: jiri.valenta@vfn.cz 


\section{Introduction}

Snakebites caused by dangerous exotic venomous snakes, which can lead to life-threatening envenoming, are rare in Europe and North America, where most victims are breeders. Due to the very low incidence of these injuries the literature consists mostly of case reports or summaries of case reports, often dating back 40 years or more, and most healthcare providers are not experienced with the management of these cases. The detailed clinical course including the early recognition of life-threatening complications and appropriate therapeutic interventions are therefore not commonly known by most physicians treating the envenomed patient.

Statistical reports on snakebites by dangerous non-native species and clinical courses of envenoming are relatively rare. In the UK there were 32 snakebites by 14 exotic species registered from 1970-1977, with two life-threatening clinical courses (Reid, 1978). Subsequently, a further 43 cases of snakebite envenomation caused by 26 species in the UK was published (Warrell, 2009). Eleven exotic snakebites treated in the ICU (intensive care unit) were recorded in Berlin from 1980-1991, three of them for severe envenoming (Köppel and Martens, 1992). In north-eastern Germany and south-eastern France 157 bites by exotic snakes were registered between 1996 and 2006 (Schaper et al., 2009). In Hungary from 1970-2006 80 cases caused by 19 species of exotic snakes were described, with one death (Malina et al., 2008). The Toxic Exposure Surveillance of the American Association of Poison Control Centres presented a yearly average of 39.9 envenomings by 77 species of exotic snakes with a total of three fatal courses in the period 1995-2004 (Seifert et al., 2007). A total of 258 snakebites involving at least 61 exotic venomous species were recorded in the US between 2005 and 2011 (Warrick et al., 2014). In the Czech Republic we registered 87 victims of exotic snakebites from 1999-2013. Twenty nine cases with systemic envenoming were treated in the ICU with ten of them considered potentially life-threatening (Valenta et al., 2014). All of these articles, however, summarized only reported snakebites. The real incidence is unknown but probably higher.

In most serious cases the envenomed victim may present in organ and/or system failure, with any or all of muscle paralysis, respiratory failure, haemodynamic instability, acute kidney injury, coagulation disorder (venom-induced consumption coagulopathy, $\mathrm{VICC}$ ) and severe local injury due to necrosis and compartment syndrome. Severe envenomings and its serious complications requiring organ and systems support should be treated in the intensive care setting (Warrell, 2009).

\section{Clinical course of severe envenoming}

\section{Signs of snakebite and envenoming}

The bite is commonly visible as "double fang" marks but may be solitary from one fang or even multiple from several fangs in a row (e.g. the viper, Bitis sp.). 
Conversely, the bite site may be minimally visible (e.g. Bungarus) and so the lack of an obvious bite site does not exclude envenomation. Not all bites, however, develop into envenoming. In approximately $10-30 \%$ of bites the snake does not inject venom (a "dry bite") (Spano et al., 2013; Valenta et al., 2014).

In most cases the first sign of envenoming is local pain and swelling, which can progress cranially on the effected limb. Subsequent lymphadenitis of the draining lymph-nodes may also occur. These signs and symptoms of localized envenoming do not always progress to systemic envenoming. With some species (e.g. Bungarus sp., Micrurus sp.) envenoming is indicated by the presence of prodromal or specific signs of systemic envenoming, with no or very few discrete local signs.

Prodromal symptoms of systemic envenoming commonly arise from autonomic dysfunction and include nausea, vomiting, headache, diarrhoea, sense of coldness or hotness, blurred vision, faintness and other non-specific signs.

The actual course of systemic envenoming is specific for each snake genera and may be complicated by allergic reactions including anaphylaxis. Severe anaphylaxis to the injected venom can develop rapidly, often much earlier than the signs and symptoms of envenoming. This may lead clinicians to miss the early signs of envenoming after the anaphylaxis has been successfully treated. Administration of horse antivenom is also associated with a risk of anaphylaxis including anaphylactic shock. Intravenous administration of hydrocortisone and antihistamines together with the dilution and slow i.v. infusion of antivenom has been recommended, although no adequately powered study has demonstrated significant benefit from the use of antihistamine premedication (Caron et al., 2009). Antivenom administration is definitive therapy in most cases of severe envenoming. Timely administration and adequate dosage of the appropriate antivenom can prevent the development of systemic symptoms of envenoming. Before administration of antivenom, due to its temporary or total unavailability, and during antivenom treatment, patients have to be treated symptomatically, sometimes with organs and systems support.

All snakebite victims should receive tetanus prophylaxis if their vaccination status is lapsed or unknown.

\section{Muscle paralysis}

Paralysis of striated muscle is a characteristic of envenomation caused by the elapid snakes (Elapidae). Curare-like neurotoxins (NTX- $\alpha$ ) with postsynaptic effects, sometimes combined with highly potent presynaptically acting enzymes, are capable of causing paralysis of respiratory muscles and fatal respiratory failure (Warrell, 1995). This paralysis typically proceeds in a cranio-caudal direction. Its initial symptoms are ptosis, ophtalmoplegia, diplopia, dysarthria, dysphagia, salivation and weakening of facial muscles. Subsequently general weakness develops with weakness of neck muscles, loss of deep tendon reflexes and eventual weakness and paralysis of respiratory muscles. Death due to asphyxia will occur unless 
respiratory support is initiated (White, 1995). Snakebites caused by some species of mambas may be further complicated by generalized muscle fasciculations (Rodriguez-Ithurralde et al., 1983).

Envenomation with a substantial quantity of NTX can cause fatal paralysis just in minutes, although it usually takes several dozen minutes, but it can rarely manifest in the interval up to 10-24 hours after snakebite (Warrell, 1995; Hung et al., 2009).

Life threatening muscle paralyzing NTX are contained mainly in the venom of elapids (Elapidae): cobras (Naja sp.) - especially Asian species, king cobra (Ophiophagus hannah), African mambas, Asian kraits (Bungarus sp.), American coral snakes (Micrurus sp.) and some Austropapuan terrestrial snakes, for example the taipan (Oxyuranus sp.). NTX are also contained in the venoms of the sea snakes. Much less toxic NTX- $\beta$ (presynaptically acting phospholipases A2, PLA2) are contained in the snake venom of other families, some subfamilies of vipers (Viperinae) and rattlesnakes (Crotalinae). Clinically apparent effects from NTX- $\beta$ may be present in envenoming from Russell's viper (Daboia russelli), South American rattlesnake (Crotalus durissus) and others. Neurotoxicity from these species is however restricted to facial muscle and general weakness. Severe respiratory muscle paralysis does not occur.

\section{Treatment}

In cases of envenoming by snakes with potent NTX, the victim should be transferred immediately to medical facilities where mechanical ventilatory support is available. If required, tracheal intubation should occur early to prevent aspiration.

The effect of the postsynaptic curare-like NTX- $\alpha$ can be decreased or eliminated by acetylcholinesterase inhibitors. To test the inhibitor's efficiency, $10 \mathrm{mg}$ of edrophonium can be administered i.v. to an adult patient, atropine $0.5 \mathrm{mg}$ i.v. should be used to block the drugs' muscarinic effects. The test is considered positive if the patient significantly improves (with a peak effect within 5 minutes) before gradually deteriorating as the drugs' effect wanes. Treatment can continue with long-acting cholinesterase inhibitors, typically neostigmine at a dose of $0.5 \mathrm{mg}$ i.v. or SC every 20 minutes (Banerjee et al., 1972). This procedure has been shown to successfully treat envenoming by the curare-like NTX- $\alpha$ of cobras, including the king cobra. However it is not effective in envenomings caused by NTX combined with presynaptically acting enzymes, such as those found in the venom of the mambas (Dendroaspis sp.), kraits (Bungarus sp.), Australian tiger snakes (Notechis sp.), taipans (Oxyuranus sp.), and some other snakes.

In mamba bite envenoming, where respiratory failure is complicated by muscle fasciculation, non-depolarizing neuromuscular blocking agents and mechanical ventilation is first line treatment.

Although paralysis due to NTX- $\alpha$ may self-reverse with time on mechanical ventilation, symptomatic treatment should not be considered an alternative 
to antivenom. Antivenom should be administered in all cases of severe envenoming.

\section{Haemodynamic instability}

Venom components affect the cardiovascular system by direct and indirect effects on the vasculature and myocardium. Toxins usually decrease vessel resistance, may cause tachy- or brady-dysrhythmias and impaired myocardial contractility (Hafeez and Majeed, 2004).

Vasodilation is caused by both direct action on the vascular system and by release of kinin-based hypotensive substances. It results in hypotension and in severe cases circulatory failure and distributive type of shock. Vasodilatory hypotension may be potentiated by fluid leakage and volume loss due to damage of endothelium and/or by haemorrhage. Hypotensive compounds are contained in the venoms of most viperids, vipers and rattlesnakes, but cannot be excluded in elapids, e.g. cobras and Austropapuan snakes (Meier and White, 1995).

Conversely, vasoconstriction resulting in systemic hypertension, coronary artery spasm and myocardial ischemia is caused by sarafotoxin present in the venom of the burrowing asps Atractaspididae (Warrell, 1995).

Specific compounds directly affecting the myocardium (cardiotoxins) can be found in the venoms of the cobras Naja sp., African vipers Bitis sp., some Austropapuan snakes and other species. These toxins can cause arrhythmias and contractility disturbances (Meier and White, 1995; Ismail et al., 2012).

\section{Treatment}

Intravenous administration of crystalloids increasing the intravascular volume is indicated in systemic hypotension, whereas the use of colloids or albumin remains controversial (Lira and Pinsky, 2014). Application of fresh frozen plasma (FFP) would provide not only volume replacement, but also a certain option to normalize potential coagulopathy. Therefore an administration of FFP can be helpful in the patients with hypotension in the presence of a haemostatic dysfunction. Blood pressure and organ perfusion can be stabilized in some cases with volume replacement only. Moreover, increased diuresis induced by volume replacement, could prevent imminent renal injury.

If hypotension persists despite volume replacement therapy, application of vasopressor support such as noradrenalin is justified. Treatment then requires invasive blood pressure (IBP) monitoring and controlled infusion rate according to blood pressure changes. Ongoing cardiac output monitoring as well as monitoring of myocardial contractility and filling by ultrasonography may be used to guide therapy more precisely. If envenoming is associated with disturbed cardiac rate, rhythm or contractility other therapeutic interventions may also be required (e.g. antidysrhythmics and inotropes).

Early administration of the correct antivenom is desirable. 


\section{Haemocoagulation disorder - VICC}

Snake venoms contain a number of toxins and toxic enzymes affecting the haemocoagulation system. These substances may interfere with vast majority of the processes in plasma coagulation system, the function of platelets (PLT) and the endothelium. The haemocoagulation active components of snake venoms cause primarily generalization of thrombin activity by thrombin-like isomers or thrombin itself, with the manifestation of consumption coagulopathy. Toxic components also create direct or indirect fibrino(geno)lysis, affect the platelets and endothelial functions. These processes result in a non-characteristic haemocoagulation disorder, VICC, ranging from clinically silent changes in laboratory parameters to significant haemorrhage, (micro)thrombosis or embolism (Lu et al., 2005; Berling and Isbister, 2015).

Serious or life-threatening conditions include organ haemorrhage (brain, lungs, retroperitoneum, and gastrointestinal tract), generalized unmanageable bleeding and intravascular formation of thrombi (e.g. coronary and mesenteric vessels, brain) (Gawarammana et al., 2009). Damage to the endothelium and formation of micro-thrombi, among others, also contribute to organ disability or failure, affecting also kidney or lungs (White, 2005).

Manifestation of VICC can start relatively late. Apparent haemocoagulation laboratory abnormities can develop as far as several hours, with no detectable clinical symptomatology in that time. When suspecting coagulation disorder, thrombo-elastography (TEG) may be useful aid to early bed-side diagnosis.

Initial laboratory findings mostly involve prolonged prothrombin and activated partial thromboplastin times (PT, aPTT), decreased fibrinogen (FBG) levels and elevated levels of fibrin degradation products (FDP), including D-dimer. Antithrombin (AT) activity and PLT count are not necessarily affected, mainly in the initial phase. Subsequent laboratory tests may show immeasurable or significantly prolonged clotting times, reduced FBG up to zero levels and a massive rise in FDPs, mostly including $\mathrm{D}$-dimers. These tests and their trends are valuable for estimating the severity of envenoming. Mild changes in laboratory coagulation parameters early after a bite do not exclude the later development of a serious coagulopathy.

Many snake venoms contain components that may cause a coagulopathy, including the vipers (Atheris sp., Bitis sp., Cerastes sp., Daboia russelli, Echis sp., Macrovipera sp.), Crotalinae subfamily (Bothrops sp., Lachesis sp., Agkistrodon sp., Crotalus sp., Trimeresurus sp.). They are also inherent in the venoms of Austropapuan elapids (Acanthophis sp., Oxyuranus sp., Notechis sp.), some Naja cobras, boigas (Dispholidus typus, Thelotornis sp.) and sea snakes (White, 2005).

\section{Treatment}

Targeted early antivenom treatment plays the key role in this type of envenoming. Repeated doses may be required because the antigenicity of some haemocoagulation effecting compounds in venom is low. 
Substitution of the clotting factors (FFP and/or FBG) may be employed whilst awaiting antivenom treatment or if the antivenom is contraindicated, but the efficacy of this therapy is controversial.

If fibrinogen levels are decreasing (indicating a consumptive coagulopathy) and the venom is not neutralized by antivenom therapy, fibrinogen replacement is unlikely to restore plasma levels but may instead "feed the fire" of the consumptive coagulopathy leading to increased (micro)thrombi formation and the generation of more fibrin degradation products, worsening the coagulopathy and bleeding. Conversely, persisting afibrinogenaemia obviously carries an increased risk of serious haemorrhage, including organ haematomas. An alternative treatment for coagulopathy may be fresh frozen plasma (FFP) (White, 2005, 2009; Maduwage and Isbister, 2014). FFP contains, in addition to fibrinogen, other coagulation factors and inhibitors and the simultaneous replacement of all these proteins may temporarily improve coagulation status. Venom components either inhibit or activate platelets. Therefore in some cases thrombocytopenia does not occur or it is only mild. Platelet infusions to correct severe thrombocytopenia may simply worsen (micro) thrombi formation, similar to FBG administration. If active bleeding with severe thrombocytopenia occurs, guidelines for PLT substitution in DIC (disseminated intravascular coagulation) should be followed (Wada et al., 2013).

Heparin administration could be considered in cases of VICC, where the predominant symptom is thrombosis or embolic complication (Wada et al., 2013). Paul et al. (2003) found that a heparin treatment regime with an initial dose of 5,000 units and further doses of 2,500 units every 8 hours, in parallel with antivenom administration, reduced mortality in patients envenomed by the saw-scaled viper (Echis carinatus) and the Russell's viper (Daboia russelli) from $26 \%$ to $19 \%$, but these results are not highly significant. However, because heparin administration will not terminate ongoing VICC and may worsen bleeding its use must be carefully considered in each case and its administration cannot be generally recommended (White, 2005).

Administration of tranexamic acid may also be efficacious in the treatment of VICC if bleeding has been worsened by fibrinolysis, particularly by increasing of plasminogen activation (Wada et al., 2013).

Treatment of last resort may be the use of plasma exchange therapy (Zengin et al., 2013). The outcome, however, is not always fully satisfying (Valenta et al., 2011).

The effectiveness of treatment should be repeatedly monitored by laboratory tests.

\section{Organ dysfunction and failure}

Acute kidney injury (AKI) frequently accompanies snakebite envenoming even if specific nephrotoxic components were not found in snake venoms. Renal insufficiency arises from myoglobinuria or haemoglobinuria secondary to rhabdomyolysis or haemolysis, the influence of destructive toxic enzymes 
on renal tissue, endothelial dysfunction and obstruction of renal capillaries by microthrombi or combination of these factors. AKI is potentiated by hypovolemia due to intravascular volume loss from redistribution or bleeding (Top et al., 2006). Oligoanuria may be the first manifestation of systemic envenoming (Valenta et al., 2008), but it may also develop later in the clinical course, sometimes even due to inappropriate treatment.

AKI cannot be excluded in envenoming by most snakes, but commonly occurs after envenomation by vipers, pit vipers (and their relatives) and rattlesnakes.

\section{Treatment}

Blood pressure support by volume replacement therapy and vasopressors to maintain increased diuresis, alkalisation of urine and timely antivenom administration may prevent the development of AKI. Early antivenom treatment could act preventively in suspicion on AKI (e.g. myo-haemoglobinuria, consumptive coagulopathy, extensive swelling). Pharmacologically resistant oligoanuria requires the use of renal replacement therapy (RRT), as in other ICU patients. The presence of ongoing VICC can cause thrombotic occlusions of the device tubing set, even together with increased tendency for bleeding. If RRT is required and heparin is contraindicated, regional anticoagulation using citrate or prostacyclin may be employed (Valenta et al., 2011).

AKI is mostly reversible if envenoming is treated adequately.

Respiratory failure (acute respiratory distress syndrome, ARDS) may occur due to toxic endothelial injury and microthrombotic obstruction of the lung capillaries. Cannot be excluded even destructive effect of toxic enzymes on lung tissue. It may also rarely occur in cases of late or ineffective antivenom treatment in severe envenomings from the viper snakes (Valenta et al., 2010, 2011).

This type of respiratory failure requires an effective, early antivenom treatment, oxygen administration and respiratory support even mechanical ventilation (Bouziri et al., 2011).

Pancreatitis as a consequence of snakebite envenoming has been reported three times (Kjellström, 1989; Valenta et al., 2010; Sagheb et al., 2011).

Liver disability can be seen either as a simple rise in liver enzymes or, rarely, as hepatocellular necrosis. The pathophysiology is likely similar to that already described in respiratory failure (Barraviera et al., 1989).

\section{Severe local disability}

Compartment syndrome with intracompartmental pressure above $30-40 \mathrm{~mm} \mathrm{Hg}$ limiting perfusion is a potentially limb threatening complication of severe 
inflammation and swelling at the snake bit site (Warrell, 1995). This syndrome may develop from two main reasons. The first is the oppression caused by swelling of the affected area, especially in tight spaces of small joints. The second reason is the intrafascial expansion of osmotic active myonecrosis caused by myotoxins injected to close intrafascial space, if large fangs penetrate subfascially (e.g. rattlesnakes, puff adder, Russell's viper).

If there is a risk of compartment syndrome, the compartment pressure should be measured and the patient monitored for the development of the syndrome. If its value reaches above $30 \mathrm{~mm} \mathrm{Hg}$, there is a possibility to attempt reducing the pressure by the limb elevation, by intravenous administration of mannitol and by repeated antivenom administration in multiple doses. This procedure may preserve the perfusion and thus need for fasciotomy. Obviously, if the compartment pressure further rises and clinical manifestation (severe pain on minimal passive extension of muscles) is present, the fasciotomy have to be performed in time. Preventive use of fasciotomy is not recommended (Gold et al., 2003).

Necroses of the skin and subcutaneous tissue are caused by venom components of some vipers, rattlesnakes and cobras, predominantly by African species. Even if the antivenom treatment does not have essential influence on the local tissue disability, its administration may reduce the extent of necrosis. Early necrectomy and debridement are indicated (Warrell, 1995, 2009).

Phlegmon or abscess can also occur in the bitten area, but primary antibiotic prophylaxis after snakebite is not recommended (LoVecchio et al., 2002). If infectious complications develop, surgical and/or antibiotic treatment is required. The infectious organisms may be gram-positive, gram-negative, anaerobes or a mixture of different species. If the bacterial agents are not known, the pervasively acting, wide spectrum antibiotics with anaerobic cover should be initiated after collection of laboratory specimens for subsequent targeted therapy (Garg et al., 2009; Chen et al., 2011).

\section{Conclusion}

All victims bitten by dangerous exotic venomous snakes should be admitted, examined and observed in appropriate healthcare facilities. Because of the possibility of life-threatening organ failure all patients with systemic envenoming should be treated in an intensive care unit with availability of antivenoms, ideally by physicians with experience in clinical toxinology.

\section{References}

Banerjee, R. N., Sahni, A. L., Chacko, K. A., Vijay, K. (1972) Neostigmine in the treatment of Elapidae bites.

J. Assoc. Physicians India 20, 503-509.

Barraviera, B., Bonjorno Júnior, J. C., Arkaki, D., Domingues, M. A., Pereira, P. C., Mendes, R. P., Machado, J. M., 
Meira, D. A. (1989) A retrospective study of 40 victims of Crotalus snake bites. Analysis of the hepatic necrosis observed in one patient. Rev. Soc. Bras. Med. Trop. 22, 5-12.

Berling, I., Isbister, G. K. (2015) Hematologic effects and complications of snake envenoming. Transfus. Med. Rev. 29, 82-89.

Bouziri, A., Khaldi, A., Hamdi, A., Bel Hadj, S., Borgi, A., Menif, K., Ben Jaballah, N. (2011) Shock, acute respiratory distress syndrome and compartment syndrome following a viper bite. Tunis. Med. 89, 217-218. (in French)

Caron, E. J., Manock, S. R., Maudlin, J., Koleski, J., Theakston, R. D., Warrell, D. A., Smalligan, R. D. (2009) Apparent marked reduction in early antivenom reactions compared to historical controls: Was it prophylaxis or method of administration? Toxicon 54, 779-783.

Chen, C. M., Wu, K. G., Chen, C. J., Wang, C. M. (2011) Bacterial infection in association with snakebite: a 10-year experience in a northern Taiwan medical center. J. Microbiol. Immunol. Infect. 44, 456-460.

Garg, A., Sujatha, S., Garg, J., Acharya, N. S., Chandra Parija, S. (2009) Wound infections secondary to snakebite. J. Infect. Dev. Ctries. 3, 221-223.

Gawarammana, I., Mendis, S., Jeganathan, K. (2009) Acute ischemic strokes due to bites by Daboia russelii in Sri Lanka - first authenticated case series. Toxicon 54, 421-428.

Gold, B. S., Barish, R. A., Dart, R. C., Silverman, R. P., Bochicchio, G.V. (2003) Resolution of compartment syndrome after rattlesnake envenomation utilizing non-invasive measures. J. Emerg. Med. 24, 285-288.

Hafeez, S., Majeed, I. (2004) Cardiac arrhythmia as presentation of snakebite. J. Coll. Physicians Surg. Pak. 14, 48-49.

Hung, H. T., Höjer, J., Du, N.T. (2009) Clinical features of 60 consecutive ICU-treated patients envenomed by Bungarus multicinctus. Southeast Asian J. Trop. Med. Public Health 40, 518-524.

Ismail, A. K., Weinstein, S. A., Auliya, M., Appareo, P. (2012) Ventricular bigeminy following a cobra envenomation. Clin. Toxicol. (Phila.) 50, 518-521.

Kjellström, B. T. (1989) Acute pancreatitis after snake bite. Acta Chir. Scand. 155, 291-292.

Köppel, C., Martens, F. (1992) Clinical experience in the therapy of bites from exotic snakes in Berlin. Hum. Exp. Toxicol. 11, 549-552.

Lira, A., Pinsky, M. R. (2014) Choices in fluid type and volume during resuscitation: impact on patient outcomes. Ann. Intensive Care 4, 38.

LoVecchio, F., Klemens, J., Welch, S., Rodriguez, R. (2002) Antibiotics after rattlesnake envenomation. J. Emerg. Med. 23, 327-328.

Lu, Q., Clemetson, J. M., Clemetson, K. J. (2005) Snake venoms and hemostasis. J. Thromb. Haemost. 3, 1791-1799.

Maduwage, K., Isbister, G. K. (2014) Current treatment for venom-induced consumption coagulopathy resulting from snakebite. PLoS Negl. Trop. Dis. 8, e3220.

Malina, T., Krescsak, L., Korsos, Z., Takacs, Z. (2008) Snakebites in Hungary - Epidemiological and clinical aspect over the past 36 years. Toxicon 51, 943-951.

Meier, J., White, J. (1995) Handbook of Clinical Toxicology of Animal Venoms and Poisons. CRC Press, Boca Raton. Paul, V., Prahlad, K. A., Earali, J., Francis, S., Lewis, F. (2003) Trial of heparin in viper bites. J. Assoc. Physicians India 51, 163-166.

Reid, H.A. (1978) Bites by foreign venomous snakes in Britain. Br. Med. J. 6127, 1598-1600.

Rodríguez-Ithurralde, D., Silveira, R., Barbeito, L., Dajas, F. (1983) Fasciculin, a powerful anticholinesterase polypeptide from Dendroaspis angusticeps venom. Neurochem. Int. 5, 267-274.

Sagheb, M. M., Scharifian, M., Moini, M., Salehi, O. (2011) Acute renal failure and acute necrotizing pancreatitis after Echis carinatus sochureki bite, report of a rare complication from southern Iran. Prague Med. Rep.

112, 67-71. 
Schaper, A., Desel, H., Ebbecke, M., De Haro, L., Deters, M., Hentschel, H., Hermanns-Clausen, M., Langer, C. (2009) Bites and stings by exotic pets in Europe: an 11 year analysis of 404 cases from Northeastern Germany and Southeastern France. Clin. Toxicol. (Phila.) 47, 39-43.

Seifert, S. A., Oakes, J. A., Boyer, L.V. (2007) Toxic Exposure Surveillance System (TESS)-based characterization of U.S. non-native venomous snake exposures, 1995-2004. Clin. Toxicol. 45, 571-578.

Spano, S., Macias, F., Snowden, B., Vohra, R. (2013) Snakebite Survivors Club: retrospective review of rattlesnake bites in Central California. Toxicon 69, 38-41.

Top, L. J., Tulleken, J. E., Ligtenberg, J. J., Meertens, J. H., van der Werf, T. S., Zijlstra, J. G. (2006) Serious envenomation after a snakebite by a Western bush viper (Atheris chlorechis) in the Netherlands: a case report. Neth. J. Med. 64, 153-156.

Valenta, J., Stach, Z., Fricova, D., Zak, J., Balik, M. (2008) Envenoming by the viperid snake Proatheris superciliaris: a case report. Toxicon 52, 392-394.

Valenta, J., Stach, Z., Svítek, M. (2010) Acute pancreatitis after viperid snake Cerastes cerastes envenoming: a case report. Prague Med. Rep. 111, 69-75.

Valenta, J., Stach, Z., Kolár̆, M. (2011) Envenoming after a snakebite from the Northeast African saw-scaled viper Echis pyramidum: Prolonged therapy upon failed treatment by antivenom. Prague Med. Rep. 112, 226-235.

Valenta, J., Stach, Z., Michalek, P. (2014) Exotic snake bites in the Czech Republic - Epidemiological and clinical aspect during 15-year period. Clin. Toxicol. (Phila.) 52, 258-264.

Wada, H., Thachil, J., Di Nisio, M., Mathew, P., Kurosawa, S., Gando, S., Kim, H. K., Nielsen, J. D., Dempfle, C. E., Levi, M., Toh, C. H. (2013) The Scientific Standardization Committee on DIC of the International Society on Thrombosis Haemostasis. Guidance for diagnosis and treatment of DIC from harmonization of the recommendations from three guidelines. J. Thromb. Haemost. 11, 761-767.

Warrell, D. A. (1995) Clinical toxicology of snakebite in Africa and the Middle East/Arabian peninsula. In: Handbook of Clinical Toxicology of Animal Venoms and Poisons. Meier, J., White, J., Pp. 433-492, CRC Press, Boca Raton.

Warrell, D. A. (2009) Commissioned article: management of exotic snakebites. QJM 102, 593-601.

Warrick, B. J., Boyer, L.V., Seifert, S. A. (2014) Non-native (exotic) snake envenomations in the U.S., 2005-2011. Toxins (Basel) 6, 2899-2911.

White, J. (1995) Poisones and venomous animals - the physician's view. In: Handbook of Clinical Toxicology of Animal Venoms and Poisons. Meier, J., White, J., pp. 9-26, CRC Press, Boca Raton.

White, J. (2005) Snake venoms and coagulopathy. Toxicon 45, 951-967.

White, J. (2009) Factor replacement for Australian snakebite coagulopathy: a re-evaluation? Intensive Care Med. 35, 1503-1054.

Zengin, S., Yilmaz, M., Al, B., Yildirim, C., Yarbil, P., Kilic, H., Bozkurt, S., Kose, A., Bayraktaroglu, Z. (2013) Plasma exchange as a complementary approach to snake bite treatment: an academic emergency department's experiences. Transfus. Apher. Sci. 49, 494-498. 